\title{
Conceptualización persona y herida para el diseño de una aplicación móvil
}

\section{Conceptualization person and injury for the design of a mobile application}

Autora: Vanessa Tiemi Furuko ${ }^{1}$

1. Enfermera Máster en Gestión y Aplicación del Conocimiento de Autocuidado en Enfermería

Recibido el: 18 de febrero de 2019

Aceptado el: 27 de febrero de 2019 


\title{
Resumen
}

La combinación de la actividad profesional del enfermero con las nuevas tecnologías, y el interés por el cuidado y tratamiento de las heridas, son el origen de este artículo. Por este motivo, se han analizado tres líneas principales en esta investigación.

En primer lugar, se analiza a la persona desde el punto de vista de las teorías de la enfermería de Dorothea E. Orem y Betty Neuman. En segundo lugar, se estudia la herida como la modificación de la estructura normal de la piel. La herida es analizada según las mismas teorías de persona anteriormente estudiadas, y se clasifican los diferentes tipos de herida conforme las clasificaciones actuales. En tercer lugar, se procede a analizar los lenguajes técnicos aplicables: desde el punto de vista enfermero y desde el punto de vista informático.

Una vez justificadas las tres líneas de investigación este estudio utiliza metodología deductiva y técnica de adquisición del conocimiento por extracción desde fuentes bibliográficas.

Los resultados obtenidos representan a la persona en su capacidad y demanda de cuidados y los distintos tipos de heridas según clasificaciones encontradas. El resultado final es el diseño de una aplicación móvil según estándar de especificación de requisitos IEEE 830 para soporte en la evaluación y tratamiento de personas con una herida de tipo úlcera por presión

Palabras claves: Aplicaciones móviles; Cuidados de enfermería; Herida

\begin{abstract}
The combination between nursery's professional activity with new technologies, and the interest on wounds' care and treatment, are the origin of this document. To achieve this purpose, three different lines have been proposed.

At first, it has been analyzed the person as per nursery theories of Dorothea E. Orem and Betty Neuman. Secondly, wounds will be evaluated as an alteration of the normal skin structure. Wounds are analyzed as per the same nursery theories abovementioned, and are listed as per current approved designations. Finally, applicable technical languages will be studied and selected for this document: from nursery perspective to computer sciences perspective.

Once those three investigation lines are justified, this survey will use the deductive methodology and the knowledge acquisition by extraction techniques from the related bibliography to achieve the expected
\end{abstract}


results.

The obtained results represent the person inside their own capacity and care demand, and the different wounds types according to existing classification. The result is the design of a mobile application, developed according IEEE 830 requirements, to support the evaluation and treatment of pressure ulcer wounded persons.

Keywords: Mobile application; Nursing care; Wounds

Tiemi Furuko, V. (2019). CONCEPTUALIZACIÓN PERSONA Y HERIDA PARA EL DISEÑO DE UNA APLICACIÓN MÓVIL. Revista ENE de Enfermería, 13(1). Consultado de http://www.ene-enfermeria.org/ojs/index.php/ENE/article/ view/964 


\section{INTRODUCCIÓN}

El avance de las ciencias relacionadas con la informática es exponencial tanto en la cantidad de aplicaciones como en la velocidad a la que evolucionan. El aumento vertiginoso del uso de la tecnología en todos los ámbitos es una realidad de nuestro día a día, donde el límite parece que se encuentra sólo en la imaginación humana. Todos los sectores de la sociedad se adaptan a ello, y de igual modo, el sector sanitario.

Estos avances de las tecnologías generan mucha inquietud profesional, es decir, producen unos deseos en los profesionales de innovar y proponer nuevas soluciones para los problemas del día a día.

Por otra parte, el área donde las principales inquietudes de la autora están enfocadas, relacionadas en su mayoría con el cuidado de las heridas, se encuentra de algún modo sin explorar suficientemente. En este momento, se planteó la pregunta en la que se sustenta el presente estudio: ¿Qué elementos de la salud describen la evolución de las heridas en la persona?

La voluntad de promover mejorías en la actividad profesional con las nuevas tecnologías, y el interés por el cuidado y tratamiento de las heridas, son el origen de este artículo y cuyos estudios, teorías fundamentadas en bases conceptuales, análisis, efectos y consecuencias se expresan en los siguientes capítulos.

La presente investigación se sustenta en el estudio de la relación entre personas y heridas, y la implementación y monitorización de esa relación a través de una aplicación para dispositivos móviles.

\section{Conceptualización de la persona}

Al tratar el concepto de persona es preciso especificar primeramente su concepto según la Real Academia de la Lengua Española (R.A.E) (23 ${ }^{\text {a }}$ Edición), define el concepto de persona como ${ }^{1}$ : 1 . f. Individuo de la especie humana. Para un entendimiento mejor en la RAE humano/a es definido como: 1. adj. Dicho de un ser: que tiene naturaleza de hombre (II ser racional). Es importante recordar que las personas pueden ser agrupadas de diferentes maneras. Por la edad, por su cultura, por su vulnerabilidad, por grupos socioeconómicos, políticos, género y etc. 
Para la psicología, una persona es alguien específico, en el concepto de la psicología abarca los aspectos físicos y psíquicos del sujeto que lo definen en función de su condición de singular y único. ${ }^{2}$ En contrapartida, la persona en el ámbito biológico es definida como un ser perteneciente a una especie concreta con su acervo genético de la especie humana. Ya en el ámbito sanitario, la persona es un ser integrado por los aspectos físicos, psíquicos y espirituales vinculados a un contexto familiar y social concreto. ${ }^{3}$

\section{La persona en la disciplina de enfermería}

Considerando la persona como un fenómeno a estudiar por la disciplina de la enfermería, fueron elegidos el modelo y teoría de enfermería de Betty Neuman y Dorothea E. Orem.

\section{Persona según el modelo de Betty Neuman}

Betty Neuman creó un modelo "basado en la teoría general de sistema y refleja la naturaleza de los organismos como sistema abierto (Von Bertalanffy, 1968) en interacción entre ellos y con el entorno (Neuman 1982)" 4,5.

En el modelo de sistemas de Neuman, propone que las enfermeras miren a la persona como un sistema holístico, con constante interacción con el ambiente que puede promover una alteración del equilibrio, y partiendo de eso promover medidas para la prevención, tratamiento adecuado y reconstitución. 4

Con base en la teoría del modelo de sistemas de Betty Neuman, la persona es definida como un sistema cliente abierto o cliente. El sistema cliente está continuamente en interacción con el entorno y estímulos estresores que pueden provocar un desequilibrio en este organismo. 4, 6,7 


\section{Persona según Dorothea E. Orem}

Según Dorothea Orem, el concepto metaparadigmático de persona se define como un ser capaz de promover el autocuidado con la connotación "por uno mismo y para uno mismo" 5 , un ser que presenta funciones biológicas, simbólicas y sociales, y con potencial para aprender a satisfacer los requisitos de autocuidado, y si no es capaz, serán otras personas las que le proporcionen los cuidados.

Además, los factores condicionantes básicos son los factores que afectan las capacidades de las personas para ocuparse de su autocuidado. ${ }^{8}$ En la igualdad de condiciones, los seres humanos tienen la capacidad de desarrollar habilidades prácticas e intelectuales que mantienen la motivación para el autocuidado y de los miembros dependientes. Donde la cultura son elementos importantes que interfieren en los requisitos de autocuidado, y son variable en los individuos y en grandes grupos. El autocuidado y el cuidado de los miembros dependientes son intencionados. 8 "El autocuidado se ve afectado no solo por la posición y roles del individuo en la familia, sino también por su estado de salud" ".

Para Orem la agencia de autocuidado es lo que movía las personas a realizar acciones, a autocuidarse, enumerando diez habilidades que promovía la persona para realizar las actividades requeridas para el autocuidado.

1) "Habilidad para mantener la atención y ejercitar vigilancia para el autocuidado tanto de factores externos e internos" 8 .

2) "Uso controlado de la energía física para iniciar y mantener las acciones del autocuidado" 8.

3) "Habilidad de controlar la posición del cuerpo para iniciar y terminar las acciones de autocuidado" 8 .

4) "Habilidad para pensar dentro de un marco de autocuidado de referencia" 8.

5) "Motivación" 8.

6) "Habilidad de toma de decisiones y hacerlo operativo" 8.

7) "Habilidad de adquirir conocimientos para el autocuidado, recordarlo y hacerlo operativo" 8.

8) "Habilidad para conocer, percibir, manipula, comunicarse e interrelacionarse para realizar el autocuidado" 8 .

9) "Habilidad para ordenar acciones de autocuidado para el autocuidado" 8.

10) "Habilidad para realizar acciones efectivas de autocuidado, integrando la vida familiar, 
personal y comunitaria" ${ }^{8}$.

Siendo estas habilidades influenciadas por los factores condicionales básicos.

Por otro lado, el déficit de autocuidado se presenta cuando la agencia de autocuidado no es suficiente para reconocer la demanda de autocuidado terapéutico, ocasionando en la persona una vulnerabilidad, que puede provocar una enfermedad, que fue definida por Orem como "proceso biológico anormal con unos sintomas característicos" 8,4

\section{Conceptualización de la Herida}

Es conveniente referenciar herida según la RAE y en el ámbito sanitario. Según la Real Academia de la Lengua Española (23 a Edición) define el concepto de herida como: 1. f. Perforación o desgarramiento en algún lugar de un cuerpo vivo. ${ }^{1}$

Las heridas son resultados visibles de la lesión o muerte de las células. A continuación, se listan los diferentes tipos de lesiones que pueden aparecer por una herida.

\section{Clasificación de los tipos de heridas}

\section{Según el grado de contaminación}

La clasificación según el grado de contaminación puede ser determinada por el contaje de colonia de bacteriana, en tejido desvitalizado el contaje con el total de $10^{6}$ colonias por grama de tejido o en tejidos necróticos con el contaje de $10^{4}$ colonias determina que la herida está infectada. Otro medio para la identificación de contaminación es por la característica del exudado. El exudado seroso y transparente está relacionado normalmente a lesiones limpias, el exudado sanguinolento indica, muchas veces, presencia de lesiones vascular y el exudado purulento es resultado de leucocitos y microrganismos vivos o muertos, presentando coloración que poden variar entre, amarillo, verde o marrón que se demuestra infección. 9, 10,11 
Sin embargo, las heridas también pueden ser clasificadas como limpias cuando no están infectadas; heridas limpias-contaminadas normalmente realizadas en el sistema respiratorio, digestivo, genital o urinario, pero no muestran signo de infección; heridas contaminadas cuando la herida muestran signos de inflamación. ${ }^{12}$

\section{Según su causa}

Las heridas pueden ser clasificadas de acuerdo a la forma en que se produjeron.:

- Heridas incisivas, hechas con un instrumento cortante, puede o no ser intencionada.

- Heridas contusas, herida cerrada como resultado de un golpe con un instrumento sin punta, donde la piel aparece equimótica.

- Heridas abrasivas, herida abierta, como resultado de una fricción.

- Heridas punzantes son heridas abiertas con un instrumento cortante que penetra en la piel y los tejidos internos. Pueden ser accidentales como en el caso de un pinchazo a la vena o intencionadas como cuando el cirujano lo hace para colocar un drenaje.

- Heridas lacerantes: Heridas que ocurre cuando los tejidos se rasgan, produciendo bordes irregulares.

- Heridas por amputación: Heridas que es resultado de la pérdida parcial de algún segmento corporal. $9,13,14$

\section{Según el espesor de los tejidos afectados}

- Epidérmicas: Heridas que afectan a solamente a la epidermis.

- Erosión: Heridas que presentan perdidas de sustancia o desprendimiento de epidermis.

- Superficiales: Heridas que presentan lesiones hasta los tejidos subcutáneos.

- Profundas, complicadas o completas: Heridas que afectan los tejidos más profundos.

- Penetrantes: Heridas que ocurren cuando un instrumento se introduce profundamente en los tejidos.

- Perforantes: Heridas que afectan a vísceras huecas albergadas en aquellas cavidades.

- Por empalamiento: Heridas causadas por un objeto inciso-punzante, de forma que el objeto queda atrapado en el cuerpo del sujeto o en alguno de los orificios naturales del organismo, provocando diferentes tipos de lesiones y afectaciones en distintos órganos que se ven afectado. $9,13,12$ 


\section{Según la dirección}

- Longitudinales: Cuando el trayecto de la herida sigue un curso más o menos paralelo al eje principal siguen un trayecto recto.

- Oblicuas: Similares a las transversales. La diferencia está en el ángulo que forma la línea imaginaria que sigue el corte de los planos de la piel causada por la herida con las líneas de adherencia de ésta.

- Transversales: Donde los bordes cutáneos de la herida no siguen las líneas de tensión de la piel.

- Espiroideas: Producidas por contusiones, la fuerza aplicada por el agente causante provoca compresión e incluso tensión en el foco traumático. ${ }^{11}$

\section{Según la forma de los bordes}

- Simples: Las heridas simples son ruptura de la continuidad cutánea limitada en profundidad al tejido graso subcutáneo, sin afectar el músculo, hueso, articulación, grandes arterias, nervios, tendones y sin pérdida importante de sustancia.

- Angulares o estrelladas: Las heridas estrelladas constituyen un desafío, ya que la gran contusión produce desvitalización de áreas de la piel afectada, que en un territorio donde ésta es escasa.

- Avulsivas: Heridas con desgarro y destrucción del tejido.

- Con pérdida de sustancia: Se produce la destrucción de todos los elementos cutáneos, epidermis, dermis e hipodermis. $9,13,12$;

\section{Según sus complicaciones}

- Simples o superficiales: En general son de buen pronóstico y suelen curar bien, sin alteración en la cicatrización. Donde solo se lesiona tejidos celulares subcutáneos.

- Profundas o complejas: Son heridas que están lesionas estructuras más complejas como vasos, nervios y músculos, generalmente tiene peor evolución y pronósticos, también suelen ser más extensas. $9,10,12$

\section{Según su evolución}

- Agudas: Las heridas agudas son aquellas que la cicatrización ocurre en el periodo esperado, las principales causas son por traumatismos, pero también pueden ser por infección, 
vasculares, alérgicas, radioactivas y térmicas.

- Crónicas: Las heridas crónicas son aquellas que no curan en un tiempo establecido, en general están asociadas a enfermedades pre-existentes como diabetes, los ejemplos más comunes son las úlceras por presión, úlceras varicosas, úlceras vasculares, úlceras neuropatías, úlceras neoplásicas. $9,13,11$

\section{Según su Profundidad}

Las heridas clasificadas por la profundidad generalmente son utilizadas para describir lesiones de quemaduras y úlceras por presión. Donde la clasificación es realizada por el tejido visualizado o por el sistema de categorías según su grado de comprometimiento de los tejidos. 91315

\section{Quemaduras}

En los casos de quemaduras ${ }^{9}$ ocurren por consecuencia de incendios, llamas, líquidos o vapores.

$1^{\circ}$ Grado: Afectación superficial (epidermis) con eritema, hiperemia, dolor e inflamación $2^{\circ}$ Grado: Pérdida de la epidermis y de la capa superficial de la dermis.

$3^{\circ}$ Grado: Pérdida total y profunda de la epidermis y dermis, caracterizada por lesión de color rojo o negro y con exposición de la grasa subcutánea.

\section{Úlceras por presión}

Las úlceras por presión son heridas ocasionadas en la piel por presión continuada. Se clasifican en categorías:

Categoría I: Eritema no blanqueable

Categoría II: Úlcera de espesor parcial

Categoría III: Pérdida total del grosor de la piel

Categoría IV: Pérdida total del espesor

Úlcera no estadiable: Sospecha de lesión de tejido profundos 15

\section{Según la integridad de la piel}

- Abierta: Heridas donde se pierde la continuidad de la piel o de las mucosas.

- Cerrada: A pesar de que la piel no está cortada, estas heridas no dejan de ser potencialmente 
dañinas, ya que existe un daño interno a los vasos sanguíneos, órganos internos, huesos, tendones o ligamentos. Hay tres clases principales de heridas cerradas: Contusiones, heridas por aplastamiento y hematomas. ${ }^{9,13}$

\section{La herida desde modelos y teorías de enfermería}

A continuación de la conceptualización de la herida por la óptica fisiológica, se estudian las heridas a través de los modelos y teorías de la enfermería de las dos enfermeras mencionadas anteriormente: Betty Neuman y Dorothea Orem.

\section{Betty Neuman}

Neuman no define herida propiamente dicha, pero podemos entender la herida como una enfermedad. Que es definida como el estado de desorganización del sistema y "aparece cuando no se satisface las necesidades y causa un estado de inestabilidad y gasto de energía" 4 , o sea un estresor al sistema.

Para entender el proceso de surgimiento de la herida en el sistema cliente vamos a considerar las heridas crónicas del tipo úlcera por presión como la enfermedad del sistema cliente. En el proceso para producir una herida crónica es posible concluir que la línea flexible de defensa fue sobrepasada, resultado de un conjunto de situaciones que llevan al desequilibrio del sistema. Esta situación será producida por la presencia de un desequilibrio en las estructuras básicas, como la mala condición actual del sistema cliente (movilidad, nutrición, actividad, percepción sensorial), también por el fallo de la prevención primaria y secundaria, por la presencia del elemento estresor. De esta forma, se obtienen así algunas variables en el sistema cliente, como la fisiológica y psicológica. Sin olvidar que las demás variables también están presentes, sea para la reconstitución del sistema cliente o para su empeoramiento. ${ }^{7}$

Por lo tanto, así como la visión holística es utilizada para el entendimiento del sistema cliente y su tratamiento, también necesitamos tener esta visión sobre el proceso de enfermedad, para promover el proceso enfermero integrado y adecuado.

\section{Dorothea E. Orem}

Según Orem "las enfermeras deben ser capaces de pensar en la enfermería, así como de realizar las operaciones de la práctica. Deben trabajar en situaciones vitales para originar condiciones benéficas para las personas a las que cuidan" 8 .

Así que, en una persona con enfermedad, por ejemplo, la herida, las enfermeras deben tener informaciones exactas de las situaciones y acontecimientos existentes en los pacientes, y de los cambios en los requisitos de autocuidado que empiezan a surgir, para una toma de decisión más 
asertiva para realizar acciones intencionadas cuanto al tratamiento de sus pacientes. Y para eso las informaciones de los factores condicionantes básicos y los requisitos de autocuidado son primordiales para una mejor elección de un tratamiento de enfermedad, lesión o incapacidad. ${ }^{8}$

\section{Lenguaje; Sistema basado en conocimiento y triangulación}

El método taxonómico es practicado desde la antigüedad en el área astronómica, la medicina, la geografía y la botánica. ${ }^{16}$ Sin embargo, la taxonomía en la salud creada muchos años después, en el ámbito enfermero culminó con la creación de la North American Nursing Diagnosis Association (NANDA) en el año 1982. La asociación surgió porque en la década de 1970 los cuidados específicos realizados por los enfermeros no podían ser identificados con autonomía, ya que no podían ser codificados. Ante esta situación, fueron realizadas reuniones científicas que proporcionaron la creación de la NANDA. ${ }^{16}$ Posibilitando un lenguaje común, que facilita el estabelecimiento de un criterio universal para todas las intervenciones y resultados, y garantiza un servicio asistencial de calidad en condiciones similares en todo el mundo.

Hoy la evolución de las taxonomías enfermero claramente está siendo realizada para mejorar la comunicación entre enfermeros.

Las normativas de todos los países incluyen ya las definiciones de las taxonomías más utilizadas en los datos que los informes clínicos deben contener obligatoriamente. En España, el Real Decreto 1093/2010 de 3 de septiembre, por el que se aprueba el conjunto mínimo de datos de los informes clínicos en el Sistema Nacional de Salud, define en sus anexos VII y VIII que los Diagnósticos Enfermeros deben clasificarse según la taxonomía NANDA, NOC y NIC, y por este motivo se escoge las dichas taxonomías para el desarrollo de este artículo. ${ }^{17}$ 


\section{Taxonomía NANDA}

La taxonomía NANDA (North American Nursing Diagnosis Association) es la principal organización para el desarrollo y perfeccionamiento de los términos para los diagnósticos enfermeros. Una sociedad científica de enfermería que busca estandarizar el diagnostico de enfermería. NANDA actualiza oficialmente su listado cada 2 años. 18; La última edición, 11²018-2020 contiene 244 diagnósticos, donde se han añadido 17 nuevos y 72 han sido revisados.

\section{Taxonomía NOC}

La taxonomía NOC (Nursing Outcomes Classification) clasificación de resultados de enfermería en castellano, presenta una terminología y unos criterios estandarizados para los resultados mensurables como consecuencia de las intervenciones realizadas por enfermeras, o sea se trata de estructurar los resultados para medir la eficacia y efectividad de los cuidados. Fue desarrollada en la University of Iowa College of Nursing desde 1991. Hoy en la $5^{\circ}$ edición contiene 490 resultados, 32 clases y 7 dominios. 19

\section{Taxonomía NIC}

La Taxonomía NIC (Nursing Interventions Classification) o clasificación de intervenciones enfermeras, utiliza las intervenciones de enfermería junto con el diagnostico enfermero, utilizando un lenguaje normalizado y global para representar los tratamientos que realizan los profesionales de enfermería. ${ }^{20}$

Las intervenciones pueden ser directas o indirectas. Directas son intervenciones efectuadas en el paciente y/o familia a través de acciones enfermeras, y las intervenciones indirectas son acciones realizadas sin el paciente, pero en beneficio del mismo o de un grupo de pacientes. 20 


\section{Sistema basado en conocimiento}

Los Sistemas Expertos o sistemas basados en el conocimiento son la parte de la Inteligencia Artificial que utiliza los conocimientos y los procedimientos de inferencia para resolver problemas altamente complejos que necesitan de gran cantidad de experiencia humana para ser resueltas. Para la resolución de estos problemas, se utilizan habitualmente sistemas informáticos. ${ }^{21}$

Con el desarrollo de la informática, estos sistemas expertos se han expandido hacia todas las áreas de conocimiento.

Los sistemas basados en el conocimiento se dividen básicamente en las siguientes partes:

- Base de conocimiento. Conjunto de todos los conocimientos introducidos, de forma que se crea una base de datos del conjunto que va a ser analizado.

- Base de Hechos. Conjunto de resultados de deducciones o inferencias, que el sistema va creando según las respuestas del usuario experto.

- Motor de Inferencia. Algoritmos que combinan la base de conocimiento con la base de hechos.

- Interfaz de usuario. La forma en la que el sistema recoge los datos. Debe ser lo más sencilla posible para que la transmisión de información entre el Motor de Inferencia y el usuario sea efectiva.

La viabilidad de un sistema basado en el conocimiento tiene su fundamento en la cantidad y calidad de las informaciones que es capaz de gestionar. Es decir, un sistema basado en el conocimiento debe buscar la mayor agilidad posible en conseguir el conocimiento, y mejorar automáticamente la calidad de la información recibida. ${ }^{21,22,23}$

Las ventajas de un sistema experto se resumen en la permanencia del conocimiento adquirido, la utilización múltiple del conocimiento experto, la eficacia constante del sistema independiente de factores externos y vitales, y la inmediatez de la solución. 21,22,23 


\section{Triangulación Taxonómica}

A nivel mundial, el método de investigación más usado y aceptado es el método científico. El método científico es un procedimiento que tiene como meta establecer relaciones entre hechos, para conseguir unas leyes que documenten el funcionamiento de estos hechos.

El método de la triangulación de datos consiste en estudiar un mismo acontecimiento usando como base por lo menos tres fuentes de datos. El objetivo de usar este método es reducir "la posibilidad de posibles incorrecciones derivadas de la falacia del razonamiento pero que en ningún caso aseguran un razonamiento válido sino una suposición lógica que debe ser contrastada." ${ }^{24}$ Aplicar este método al área de la enfermería, tiene la intención de analizar cualquier enfermedad o asunto relacionado con la enfermería desde varios puntos de vista.

La Triangulación Taxonómica es una evolución del método científico. Este método consiste en vigilar continuamente las valoraciones, los objetivos y criterios de resultados (NOC) y las intervenciones y actividades realizadas (NIC) en un mismo paciente, de forma que en todos los procesos se realiza una comparación con el Diagnóstico (NANDA). Este análisis posterior quiere comparar el diagnóstico estimado con la evolución del resto de parámetros. Así se comprueba si la elección del diagnóstico NANDA ha sido correcta. 25,24

\section{Implementación por medio de una aplicación móvil}

Con la evolución de la tecnología, actualmente se puede tener una infinidad de información accesible solamente tocando una pantalla. El objetivo es demostrar que es posible acceder a esta información de forma sencilla y común para gran parte de las personas. Será usada una aplicación móvil como interfaz por ser, ahora mismo, la forma más simple para comunicar una base de datos de un sistema experto con un usuario con conocimientos medios sobre las heridas. 


\section{METODOLOGÍA}

La metodología empleada para el estudio es de tipo deductivo. Se utiliza mediante técnica de extracción de conocimiento, a partir de búsqueda bibliográfica, análisis documental y educción de expertos.

Para conseguir el objetivo general, es necesario alcanzar los diferentes objetivos específicos indicados previamente.

A seguir se describen las metodologías utilizadas para cada uno de los objetivos específicos.

\section{Metodología para describir los conceptos de persona a través del modelo de cuidado de Orem y Neuman}

Para la realización de la metodología para el objetivo uno fue de tipo deductivo con extracción de conocimiento, a partir de búsqueda bibliográfica, análisis documental y educción de expertos.

La metodología para el objetivo específico uno, es describir los conceptos de persona a través del modelo de cuidado de Orem y Neuman y fue realizada con las siguientes fases: Primera fase, se utilizó la extracción de conocimiento de las teorías de diferentes disciplinas al respecto de la persona. En este primer objetivo, se analizan las diferentes teorías de persona que se han desarrollado durante la historia, pesquisando además teorías que pudieran ser de interés en las diversas disciplinas presentes.

Segunda fase Una vez establecidas las bases que sustentan el primer objetivo, se inicia el estudio de las bases teóricas en enfermería y correlacionando el concepto persona.

Para ese objetivo las teóricas en enfermería elegidas fueron: Betty Neuman ${ }^{7}$ y Dorothea E. Orem ${ }^{8}$ 


\section{Metodología para describir las tipologías de las heridas}

Se realiza con el mismo método de estudio, de tipo deductivo con extracción de conocimiento, a partir de búsqueda bibliográfica, análisis documental y educción de expertos.

Para conseguir este objetivo, fueron realizados siguiendo las fases:

Primera fase: Para conseguir este primer objetivo, se analizan las diferentes conceptualizaciones de heridas y se realizan una clasificación de los diferentes tipos de heridas, con el objetivo de conocer las tipologías existentes.

Segunda fase: Una vez analizadas las tipologías existentes, se correlaciona el concepto de herida según las teorías de Betty Neuman y luego según la teoría de Dorothea E. Orem.

\section{Metodología para el diseño de una aplicación para la evaluación de personas con heridas en el campo del cuidado}

En el objetivo específico, se hizo un diseño de una aplicación para la evaluación de personas con heridas en el campo del cuidado. Con el mismo método de estudio, de tipo deductivo con extracción de conocimiento, a partir de búsqueda bibliográfica, análisis documental y educción de expertos.

A continuación del estudio, fueron realizadas las siguientes fases:

Primera fase: Para conseguir el objetivo tres, primero se realizó un estudio de la evolución de las taxonomías enfermeros, en este apartado fueron elegidos las siguientes taxonomías enfermero: NANDA, NOC, NIC 18, 19, 20

Segunda fase: Para entender los conceptos básicos, la segunda fase se inicia con el estudio de la inteligencia artificial con su definición y paradigma. Después, fueron estudiadas las características de un sistema basado en conocimiento y, en segundo término, se realizó la construcción del SBC y por último la metodología de un SBC. Finalizada la segunda fase, fue escogida para la representación del SBC la triangulación taxonómica. Donde en esta fase se describe el concepto de triangulación y su método.

Tercera fase: En una tercera fase se procede al desarrollo de la definición de los requisitos y aplicación de diseño y de flujo de trabajo para construir la app. En esa fase se utilizó el documento 
“Especificación de Requisitos según el estándar IEEE 830". Para la ejecución de la Especificación de Requisitos según el estándar IEEE 830 se eligió las heridas de úlceras por presión.

\section{RESULTADOS}

A continuación, se muestra los resultados obtenidos en el presente artículo de investigación. Cada uno de los resultados que se presentan fueron obtenidos a partir de cada objetivo específico por lo que se monitoriza detallando cada uno.

\section{Resultado 1 describir los conceptos de persona a través del modelo de cuidado de Orem y Neuman}

Como fue expuesto en el apartado 2. Conceptualización de la persona se pueden describir a las personas por diversas disciplinas y a través de múltiples ópticas diferentes.

Betty Neuman al definir la persona como un sistema afirma que la misma está continuamente en interacción con el entorno y los estímulos estresores que pueden provocar un desequilibrio en este organismo (Figura 1). 


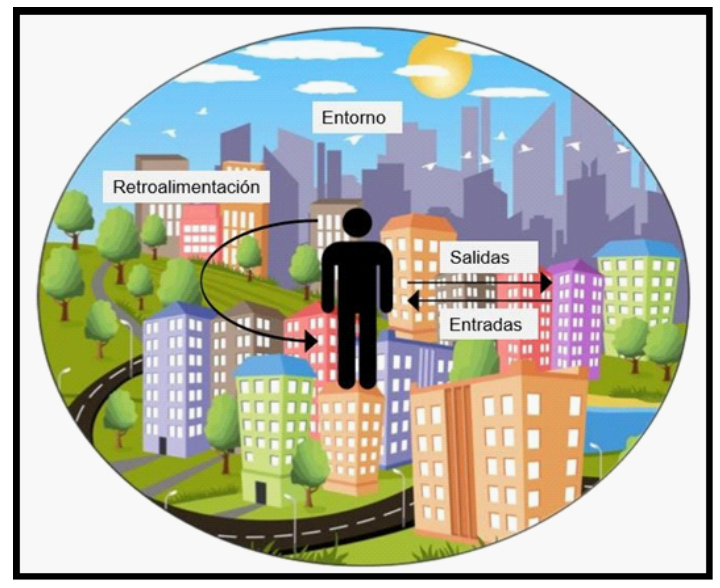

Figura 1: Representación gráfica de la persona como sistema

Fuente: Elaboración propia con inspiración en Alligood, M. R. (2014). Modelos y teorías en enfermería

Por su parte, Dorothea E. Orem define las personas como un organismo biológico, racional y pensante, como ella es afectada por su entorno y como sus acciones afectan a ella misma, a otros y a su entorno. O sea, define la persona según sus factores condicionantes básicos (FCB). Como se han visto anteriormente, los FCB son identificados como: edad; sexo; estado de desarrollo; orientación sociocultural; genero; factor de sistema de cuidado de salud; estado de salud; factores del sistema familiar; factores ambientales y recursos. Y en cada FCB se incluyen las demandas y agencias de autocuidado (Figura 2).

\begin{tabular}{|c|c|c|c|c|c|c|}
\hline & DM & AG & DM & AG & DM & $A G$ \\
\hline Edad & \multicolumn{2}{|c|}{$0-20$ años } & \multicolumn{2}{|c|}{21 - 64 años } & \multicolumn{2}{|c|}{$\geq 65$ años } \\
\hline Sexo & $\uparrow \uparrow$ & $\downarrow \downarrow$ & $=$ & $=$ & $\downarrow$ & $\downarrow$ \\
\hline $\begin{array}{l}\text { Estado de } \\
\text { desarrollo }\end{array}$ & $\uparrow \uparrow$ & $\downarrow \downarrow$ & $=$ & $=$ & $\downarrow$ & $\downarrow$ \\
\hline Género & $\uparrow \uparrow$ & $\downarrow \downarrow$ & $=$ & $=$ & $\downarrow$ & $\downarrow$ \\
\hline $\begin{array}{l}\text { Orientación } \\
\text { sociocultural }\end{array}$ & $\uparrow \uparrow$ & $\downarrow \downarrow$ & $=$ & $=$ & $\downarrow$ & $\downarrow$ \\
\hline $\begin{array}{l}\text { Factores del } \\
\text { sistema de } \\
\text { cuidado de la } \\
\text { salud }\end{array}$ & $\uparrow \uparrow$ & $\downarrow \downarrow$ & $=$ & $=$ & $\downarrow$ & $\downarrow$ \\
\hline $\begin{array}{c}\text { Factores del } \\
\text { sistema familiar }\end{array}$ & $\uparrow \uparrow$ & $\downarrow \downarrow$ & $=$ & $=$ & $\downarrow$ & $\downarrow$ \\
\hline Estado de Salud & $\uparrow \uparrow$ & $\downarrow \downarrow$ & $=$ & $=$ & $\downarrow$ & $\downarrow$ \\
\hline $\begin{array}{c}\text { Factores } \\
\text { ambientales }\end{array}$ & $\uparrow \uparrow$ & $\downarrow \downarrow$ & $=$ & $=$ & $\downarrow$ & $\downarrow$ \\
\hline Recursos & $\uparrow \uparrow$ & $\downarrow \downarrow$ & $=$ & $=$ & $\downarrow$ & $\downarrow$ \\
\hline
\end{tabular}


Figura 2: Análisis de la agencia y demanda de autocuidado según factores condicionantes básicos Fuente: Elaboración propia. Leyenda: DM: Demanda de Autocuidado; AG: Agencia de Autocuidado

Siguiendo el modelo de déficit de autocuidado de Dorothea E. Orem para describir las personas conforme sus factores condicionales básicos (FCB), en el presente artículo se van a agrupar las personas por franja de edad. La población será dividida en 3 grupos, el primer grupo será compuesto por individuos entre 0-20 años, el segundo grupo de 21-64 años, y el tercer grupo será compuesto por individuos de 65 años y mayores.

Al realizar la división poblacional en la tabla se comparará la agencia y demanda de los grupos de acuerdo con sus FCB, el segundo grupo será considerado la normalidad, y los dos grupos con la franja de edad anterior y posteriormente la normalidad será comparada a ella.

En la franja de edad de 0-21 años, comparada con la normalidad 21-64 años, presenta las demandas de autocuidado terapéuticos aumentado en todos los FCB, y en la agencia de autocuidado terapéutico disminuido. Es decir, en esta franja de edad las competencias, que todavía están en desarrollo se representan con dos flechas hacia abajo, y sus necesidades altas, se representa con dos flechas hacia arriba. En este grupo con el pasar del tiempo sus competencias van aumentando y sus necesidades pasan a ser cada vez menores. Por tanto, este es un periodo en que la vulnerabilidad disminuye.

Para el presente análisis la franja de edad de 21-64 años, representa el grupo que posee competencias para sus necesidades, donde la demanda de autocuidado terapéutico y la agencia de autocuidado terapéutico para todos los FCB son representados con el símbolo de igual, es decir, en esa franja las personas presentan unas competencias capaces de suplir sus necesidades, por lo tanto, es la franja de edad que presenta menorvulnerabilidad.

Ya en la franja de edad mayor o igual a 65 años comparada con la franja de edad de 21-64 años, presenta las demandas de autocuidado terapéuticos disminuido con el símbolo de flecha hacia abajo una flecha hacia abajo, y las agencias de autocuidado terapéuticos también disminuido con el símbolo flecha hacia abajo. Es decir, sus necesidades con el pasar de los años van disminuyendo y sus competencias por el proceso de envejecimiento también se disminuye. Un periodo en que la vulnerabilidad es creciente con el proceso de envejecimiento.

Por lo tanto, en cada proceso vital las personas presentan una demanda y una agencia de autocuidado distintos. 


\section{Resultado 2 describir las tipologías de las heridas}

A continuación, la descripción de las personas es de suma importancia para la evaluación de una herida. Donde en este apartado las heridas serán observadas en los mismos grupos de personas, divididos en franjas de edad.

A seguir, en la imagen adjunta fue realizando un análisis de las distintas posibilidades de una misma herida afectaran a las personas en diferentes etapas vitales, identificaremos 3 casos diferentes. Para el análisis la herida elegida fueron las úlceras por presión categoría 4 en región sacra (Figura 3).

\begin{tabular}{|c|c|c|c|c|c|c|c|c|c|c|c|}
\hline & & \multirow[t]{2}{*}{ Aire } & \multirow[t]{2}{*}{ Agua } & \multirow[t]{2}{*}{ Alimento } & \multirow[t]{2}{*}{ Eliminación } & \multicolumn{2}{|c|}{ Actividad y Descanso } & \multicolumn{2}{|c|}{$\begin{array}{c}\text { Soledad e Interacción } \\
\text { Social }\end{array}$} & \multirow[t]{2}{*}{$\begin{array}{l}\text { Prevención } \\
\text { del Peligro }\end{array}$} & \multirow[t]{2}{*}{ Normalidad } \\
\hline & & & & & & Actividad & Descanso & Soledad & Interacción & & \\
\hline \multirow{2}{*}{$\begin{array}{l}0-20 \\
\text { años }\end{array}$} & DM & $=$ & $\uparrow$ & $\uparrow$ & $\uparrow$ & $=$ & $\uparrow$ & $=$ & $=$ & $\uparrow$ & $\uparrow$ \\
\hline & AG & $=$ & $=$ & $=$ & $=$ & $\downarrow$ & $\downarrow$ & $=$ & $\downarrow$ & $\downarrow$ & $\downarrow$ \\
\hline \multirow{2}{*}{$\begin{array}{l}21-64 \\
\text { años }\end{array}$} & DM & $=$ & $\uparrow$ & $\uparrow$ & $\uparrow$ & $=$ & $\uparrow$ & $=$ & $=$ & $\uparrow$ & $\uparrow$ \\
\hline & AG & $=$ & $=$ & $=$ & $=$ & $\downarrow$ & $\downarrow$ & $=$ & $\downarrow$ & $\downarrow$ & $\downarrow$ \\
\hline \multirow{2}{*}{$\begin{array}{c}\geq 65 \\
\text { años }\end{array}$} & DM & $=$ & 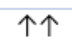 & 个个 & 个个 & $=$ & 个个 & $=$ & $=$ & $\uparrow \uparrow$ & 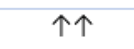 \\
\hline & AG & $=$ & $=$ & $=$ & $=$ & $\downarrow \downarrow$ & $\downarrow \downarrow$ & $=$ & $\downarrow \downarrow$ & $\downarrow \downarrow$ & $\downarrow \downarrow$ \\
\hline
\end{tabular}

Figura 3: Análisis de la agencia y demanda según Requisitos de Autocuidado Universales

Fuente: Elaboración propia

En el análisis a continuación, las heridas serán analizadas por el modelo de déficit de autocuidado de Dorothea E. Orem, porque los estresores del sistema propuesto por Betty Neuman coinciden con los procesos vitales de la teoría enfermera de déficit de autocuidado propuesto por Orem.

Empezando el análisis por la franja de edad 0-20 años la capacidad de las personas para satisfacer los distintos requisitos de autocuidado universales se ve disminuida en la agencia de autocuidado, representada por flecha hacia abajo, en los requisitos de actividad y descanso, interacción social, prevención de peligro y normalidad. Por otro lado, la demanda de autocuidado, representado por una flecha hacia arriba, esta aumentada en los requisitos de autocuidado en el mantenimiento del aporte de agua y alimento, provisión de cuidado asociados con los procesos de eliminación, mantenimiento del equilibrio del descanso, prevención del peligro y normalidad.

No obstante, en esa etapa vital el tiempo de la curación de procesos viales como en el caso de la recuperación de las heridas es más rápida que en el caso de los adultos o personas mayores. Esto 
acontece porque en la primera etapa vital, presenta una mayor velocidad de curación.

En la franja de edad de 21-64 años. En esta situación, la capacidad para satisfacer los distintos requisitos de autocuidado universales se ve igualmente disminuida en la agencia de autocuidado, representada por flecha hacia abajo, en los requisitos de actividad y descanso, interacción social, prevención de peligro y normalidad. Y la demanda de autocuidado, representado por una flecha hacia arriba, esta aumentada en los requisitos de autocuidado en el mantenimiento del aporte de agua y alimento, provisión de cuidado asociados con los procesos de eliminación, mantenimiento del equilibrio del descanso, prevención del peligro y normalidad.

Por último, en la franja de edad mayor de 65 años, la capacidad de las personas en esa franja de edad para satisfacer los distintos requisitos de autocuidado universales se ve disminuida en la agencia de autocuidado, representada por dos flechas hacia abajo, en los requisitos de actividad y descanso, interacción social, prevención de peligro y normalidad. Por otro lado, la demanda de autocuidado, representado por dos flechas hacia arriba, esta aumentada en los requisitos de autocuidado en el mantenimiento del aporte de agua y alimento, provisión de cuidado asociados con los procesos de eliminación, mantenimiento del equilibrio del descanso, prevención del peligro y normalidad.

A pesar de ser los mismo RAU en las franjas de edad anteriores, en todos los requisitos se presenta con mayor grado de tanto en la agencia cuanto en la demanda de autocuidado. Para una ejemplificación se discutirá el RAU prevención del peligro, en la cual, en esa franja de edad por el proceso de envejecimiento provocados por factores intrínsecos, la disminución de células desde el punto de vista inmunitario, pérdida de melanocitos, colágeno, producción de vitamina $\mathrm{D}$, la reducción de las glándulas sudoríparas y sebáceas, que puede resultar en deshidratación, sequedad casi permanente dejan la piel más fina, frágil, más permeable, menos elástica, por lo tanto, la agencia se presenta disminuida con mayor grado en esa población.

Por esos factores de envejecimiento y muchos otros la misma herida en las personas de esa franja de edad tiene la tendencia de tardar más para la curación que en las franjas anteriores.

Como esa representación podemos decir que en cada uno de los ochos requisitos de autocuidado universal, desde varias perspectivas, presenta de forma diferenciada para las personas con las distintas franjas de edad, sexo, estado de desarrollo, estado de salud, orientación sociocultural y recursos. Por lo tanto, un mismo proceso vital afecta las personas de un modo distinto desde la infancia hasta la ancianidad. 


\section{Resultado 3 diseño de una aplicación para la evaluación de personas con heridas en el campo del cuidado}

A continuación, se describe gráficamente el diseño orientativo de una aplicación para la evaluación de personas con heridas en el campo del cuidado. Para el diseño de la aplicación se eligió las úlceras por presión, no obstante, la aplicación es completamente funcional para el estudio de la evolución de cualquier tipo de herida (Figura .4)

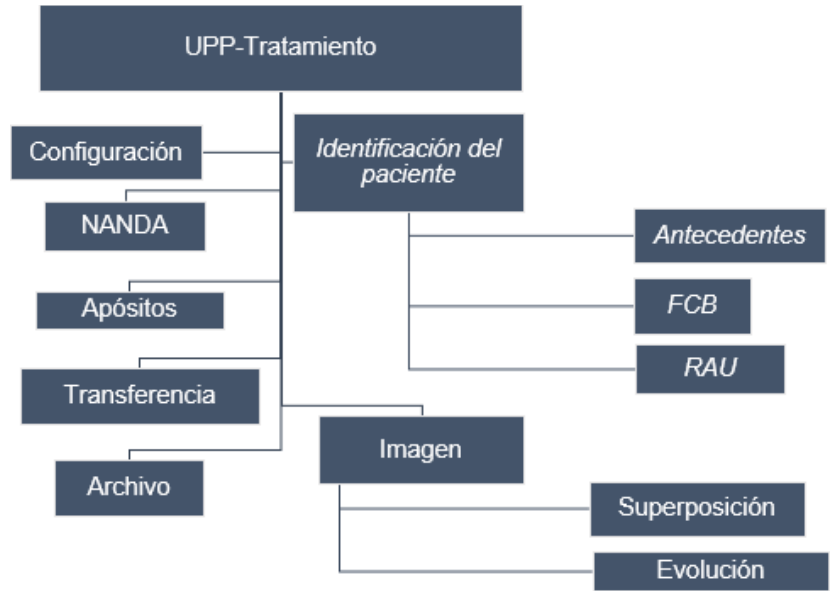

Figura 4: Esquema funcional de la aplicación

Fuente: Elaboración propia

A continuación, se incluyen imágenes orientativas del diseño pensado para esta aplicación móvil (igirara $\underline{5}$. 


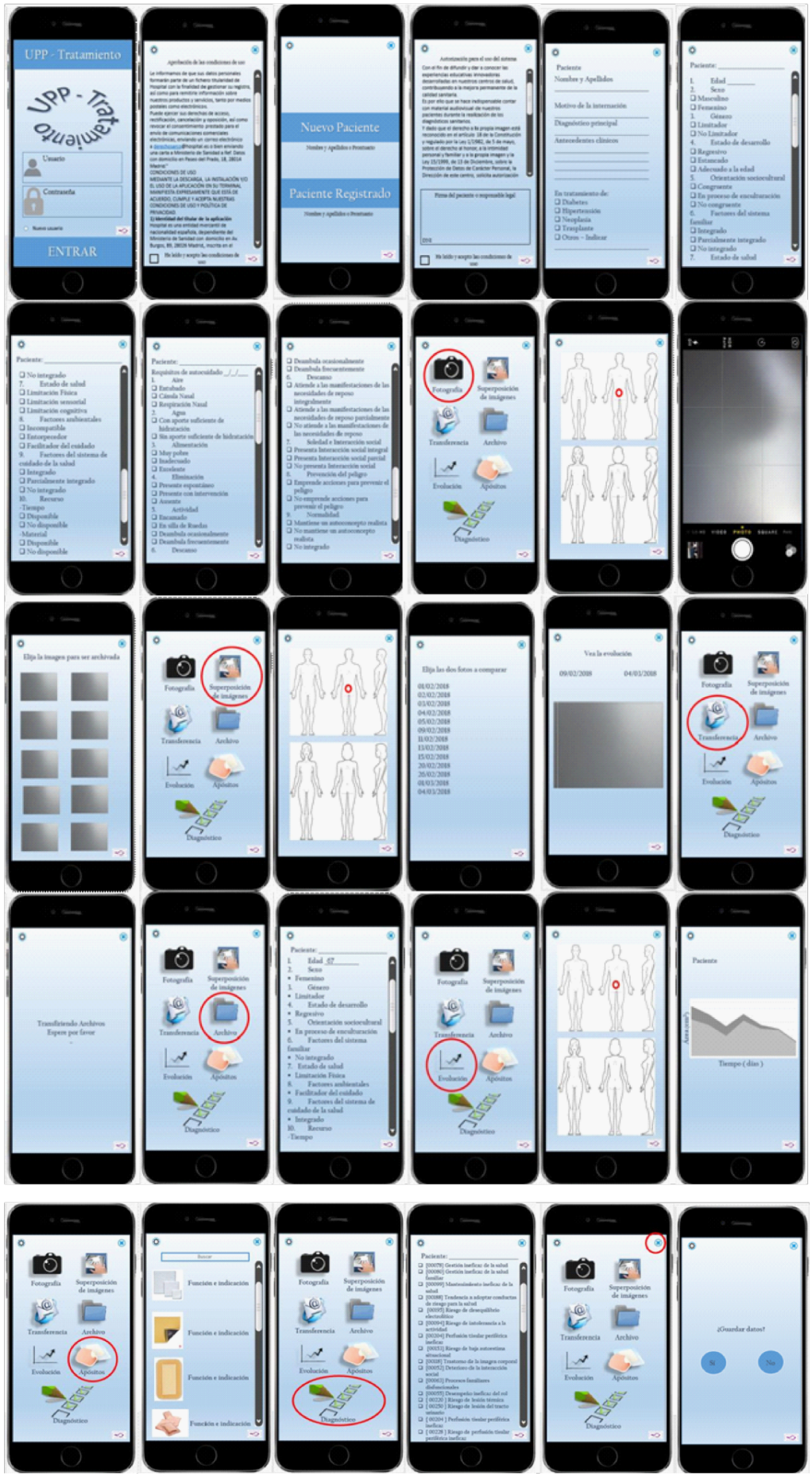

Figura 5 : Imágenes orientativas de la aplicación para úlceras por presión Fuente:

Elaboración propia 


\section{DISCUSIÓN}

Con relación al enfoque realizado en este artículo fin de máster no fue encontrado ningún trabajo publicado hasta la realización de la búsqueda bibliográfica.

A pesar de no encontrar documentos con el mismo enfoque, se tiene conocimiento de algunos trabajos de diseño en el campo de las aplicaciones relacionadas con las úlceras por presión y heridas. En su mayoría se trata de aplicaciones para la prevención y tratamiento de las úlceras por presión desde un enfoque de la patología.

Las aplicaciones encontradas no están hechas teniendo en cuenta el cuidado de las personas, en su mayoría están basadas en el tratamiento de las heridas, utilizando como base únicamente la escala de BRADEN.

Para cerrar este apartado la descripción de los conceptos de persona a través del modelo de cuidado de Orem y Neuman conjuntamente con la descripción de las tipologías de las heridas posibilitarán analizar la relación entre persona y herida.

\section{CONCLUSIONES}

Del presente artículo se puede concluir que la hipótesis planteada, que la relación entre persona y herida puede ser implementada y monitorizada a través de una aplicación para dispositivos móviles, está confirmada.

Para llegar a dicha conclusión primero fue realizada la descripción de los conceptos de persona a través del modelo de cuidado de Orem y Neuman y luego la descripción de las tipologías de las heridas, a través de eso se realizó un diseño de una aplicación para la evaluación de personas con heridas en el campo del cuidado.

En el diseño de la app, la identificación de las personas fue realizada a través de los factores condicionantes básicos conjuntamente con los requisitos de autocuidado, los antecedentes clínicos, motivo de atención, diagnóstico de cuidado y la realización de algunos tratamientos específicos, como diabetes, hipertensión, trasplante y neoplasias. En el momento de esa identificación en la app, los diagnósticos de cuidados serán seleccionados para la ayuda y soporte en la diagnosis y tratamiento terapéuticos para la utilización de los enfermeros. En este sentido sería un soporte a los enfermeros 
para la valoración continuada dentro del proceso enfermero, y posteriormente establecer una terapéutica de las heridas desde los cuidados de enfermería, con evaluación por el sistema de fotocomparación.

Por todo eso el presente artículo demuestra el objetivo general propuesto de diseñar una aplicación móvil para el cuidado de las heridas, lo que aúna los conocimientos del cuidado de las personas con las heridas, junto con las nuevas tecnologías en beneficio de la calidad de la asistencia sanitaria.

\section{LÍNEAS FUTURAS DE TRABAJO}

Como se menciona en el inicio, el límite de la tecnología informática es la imaginación humana, por lo que las posibilidades de futuras líneas de investigación de la aplicación móvil propuesta son ilimitadas. No obstante, los aspectos éticos siempre serán una cuestión que se tendrán en cuenta en el impacto de la tecnología en las personas.

En base a las conclusiones expuestas, las líneas futuras de investigación que se plantean para un desarrollo posterior son divididas en dos grandes grupos, la evolución conceptual en nuevos estudios y la evolución del uso de la tecnología en el ámbito de la salud, que serán expuesto abajo:

Profundizar en la conceptualización de la relación persona y herida.

Promover nuevos estudios para verificar el impacto de las distintas heridas en función de la edad. Por ejemplo, en los niños que presentan cuidado dependiente, teniendo en cuenta la familia y el proceso de crecimiento.

Estudio sobre los diferentes tipos de heridas con intención de unificar las diversas clasificaciones encontradas hasta la fecha.

Ampliar la app diseñada desde el método de triangulación taxonómica para el estudio de la valoración, diagnosis, planificación y tratamiento de heridas. Además, esta aplicación permitiría conocer los diagnósticos de enfermería que están implicados en el tratamiento realizado con los datos que recoge la herramienta.

Buscar nuevas funcionalidades para la aplicación móvil y considerar nuevos puntos de vistas del software.

Desarrollar la app para un estudio de pilotaje, verificar la satisfacción de los usuarios en su utilización y validar la aplicación. 
Plantear nuevas aplicaciones utilizando el mismo modelo de la relación persona y herida para buscar nuevas tecnologías para funcionalidad en el cuidado. 


\section{BIBLIOGRAFÍA}

1. Real Academia Española, «Diccionario de la Lengua Española,» 2017. En línea. Available: http:// dle.rae.es.

2 N. Symington, A Psicologia da Pessoa, Blucher, 2017.

3 D. Perestrello, A medicina da Pessoa, Atheneu, 2017.

4 M. Alligood, Modelos y Teorías en Enfemería, Elsevier, 2014.

5 M. Prado Laguna, Fundamentos Teóricos y Cuidados Básicos de Enfermería, Síntesis, 1996.

6 B. Neuman y J. Fawcett, The Neuman Systems Model, Pearson, 2011.

7 Neuman Systems Model, «Neuman Systems Model,»En línea;. Available: https:// www.neumansystemsmodel.org/new-page-1.

8 D. E. Orem, Orem Model: Concepts of Nursing in Practice, Barcelona: Masson-Salvat, 1993.

9 G. Irion, Feridas: Nova Abordagens, manejo clinico e atlas em cores, Río de Janeiro: Guanabara Koogan, 2012.

10 S. Cortés, «O tratamento de ferida: umartigo de revisao,» Cien Sena Aires, vol. 2, nº 1, pp. 55-64, 2013.

11 R. García González, M. Fornel, S. López y V. Valdés, «Abordaje de enfermería en heridas de urgencias,» Gerokomos, vol. 3, nº 24, 2013.

12 J. Martínez Rodríguez y R. Bugarín González, «Las heridas superficiales.,» Medicina Integral, vol. 35, nº 4, pp. 137-148, 2000.

13 K. Cohen, R. Diegelman y R. Dome, Wound care and wound healding, McGraw-Hill, 1999.

14 C. DEaley, Cuidado de Feridas, Sao Paulo: Atheneu, 2008.

15 National Pressure Ulcer Advisory Panel, Prevención y tratamiento de las úlceras por presión. Guía de consulta rápida, 2014.

16 S. Ortiz, Modelos de Cuidados en Enfermería: Nanda, Nic, Noc , McGraw-Hill, 2015.

17 Boletín Oficial del Estado, Real Decreto 1093/2010 de 3 de septiembre por el que se aprueba el conjunto mínimo de datos de los informes clínicos en el Sistema Nacional de Salud, 2010.

18 Nanda International, Nursing Diagnoses 2015-17: Definitions and Classification, 2014.

19 S. Moorhead, M. Johnson, M. Maas y E. Swanson, Nursing Outcomes Classification (NOC), $5^{\text {a }}$ ed., Elsevier, 2013.

20 G. Bulechek , Classificaçao das Intervençoes de Enfermagem (NIC), Elsevier Health Sciences, 2011.

21 J. M. Santamaría García, Investigación Deductiva, representación lógica e implementación computacional sobre las limitaciones de acción del autocuidado según el modelo de Dorothea Orem, Alcalá de Henares: Universidad de Alcalá, 2008.

22 M. L. Jiménez Rodríguez, Sistema basado en conocimiento para la ayuda en el diagnóstico del cansancio en el desempeño del rol del cuidador, Alcalá de Henares: Universidad de Alcalá, 2006.

23 M. Baiador Ferreras y J. Alonso Fernández, Nuevas técnicas de modelado orientado a objetos e implementación de un generador de sistemas basados en conocimiento, Madrid: Universidad Complutense, 2000 .

24 A. González Aguña, Red asociativa de los lenguajes normalizados del cuidado: Base para la Triangulación Taxonómica a propósito de la Gripe A/H1N1, Alcalá de Henares: Universidad de Alcalá, 2013.

25 A. González Aguña y J. M. Santamaría García , «El ciclo del cuidado: el modelo profesional de cuidado desde el enfoque del pensamiento,» ENE Revista de Enfermería, vol. 9, nº 1, 2015. 